\title{
Membrane Protein-GroEL Co-crystallization on Lipid Monolayers
}

\author{
Jingchuan Sun ${ }^{*}$, Christos G. Savva*, and Andreas Holzenburg ${ }^{* * * *}$ \\ *Microscopy and Imaging Center (MS 2257), and Department of Biology, **Department of \\ Biochemistry and Biophysics, Texas A\&M University, College Station, TX77843-2257, USA.
}

The structural determination of biological macromolecules by electron crystallography requires well ordered two-dimensional (2D) crystals. In recent years the lipid monolayer technique as one of the more promising strategies to grow $2 \mathrm{D}$ crystals, has been modified to accommodate transmembrane proteins [1]. The lipid monolayer technique (cf. [2] and refs. therein) is based on the ability of phospholipids to spread at the air/water interface to form a monolayer with the lipid head groups pointing towards the water and the aliphatic chains towards the air. Using modified lipid head groups that specifically bind the target protein, protein molecules in aqueous solution can be attracted to and concentrated at the lipid surface. This can lead to a local super saturation of the protein from initially dilute solutions (e.g. less than $0.1 \mathrm{mg} / \mathrm{ml}$ ) to more than $50 \mathrm{mg} / \mathrm{ml}$ at the membrane surface. Together with the degrees of rotational and translational freedom the lipid and protein molecules are experiencing, this facilitates the formation of $2 \mathrm{D}$ crystals.

Here we report the utilization of the standard lipid monolayer technique with membrane proteins using GroEL as a non-destructive (with regards to the lipid monolayer) detergent substitute. GroEL is a well-studied molecular chaperonin that is able to accommodate proteins with exposed hydrophobic surfaces. Recent in vitro experiments have shown that several membrane proteins, including bacteriorhodopsin (BR), LacY and the bacteriophage $\lambda$ holin (S protein), can be kept in solution in the presence of GroEL after removing detergent [3-4] and single particle reconstructions of GroEL-membrane protein complexes suggested that membrane protein are bound to one of the central cavities of GroEL [5]. Two of the membrane proteins (LacY and S protein) were expressed with a His $_{6}$-tag, and therefore the possibility of crystallizing membrane proteins while bound to WT tetradecameric GroEL using the lipid monolayer technique in conjunction with DOGS-NTA-Ni lipid has been investigated. The setup of the crystallization trials and specimen mounting essentially followed the protocol described by Poglitsch et al.[6]. While His ${ }_{6}$-tagged LacY- and S proteinGroEL complexes readily formed 2D crystals (Fig.1A), WT BR-GroEL complexes and WT GroEL by itself did not. This suggests that LacY and S protein can still bind to the ligand lipid while in complex with GroEL. The Fourier transform shown in Fig. 1B reveals reflections up to the $5^{\text {th }}(h, 0)$ and $8^{\text {th }}$ order $(0, k)$, respectively, as well as systematic absences that hint to the presence of a glide plane. It is reassuring that even with no symmetry imposed, the projection map highlights the 7 fold rotational symmetry of GroEL when viewed face-on, and, in addition, a central density is observed (Fig. 1C). The projection maps calculated in p2 (Fig.1D) and pg (to account for the systematic absences observed along $k$; not shown) display essentially identical features with slightly improved $\mathrm{s} / \mathrm{n}$ ratios owing to the additional symmetry operations. The density in the middle of the GroELLacY complex was verified by single particles analysis and 3D reconstruction (Fig. 1E-F) and shown to occupy one cavity only [5].

In conclusion, $\mathrm{His}_{6}$-tagged membrane proteins can be crystallized on a DOGS-NTA-Ni ligand lipidcontaining monolayer when complexed with GroEL. The membrane protein densities inside GroEL lack any discernable features, which is probably due to symmetry mismatch and orientational freedom [cf.5]. However, GroEL-membrane protein co-crystallization could become a valuable tool, if one was able to lock the cargo protein into a single position relative to GroEL. 


\section{References}

[1] D. Lévy et al., J. Struct. Biol. 127 (1999) 44.

[2] M. Hoppert and A. Holzenburg, pp.201-286 in M. Hoppert, Microscopic Techniques in Biotechnology, Wiley-VCH, Weinheim, 2003.

[3] J. Deaton et al., Proc. Natl. Acad. Sci. U.S.A. 101 (2004) 2281.

[4] J. Deaton et al., Protein Sci.12 (2004) 1778.

[5] J. Sun et al., Arch. Biochem. Biophys. 434 (2005) 352.

[6] C.L. Poglitsch et al., Cell 98 (1999) 791.

[7] The authors thank Ry Young and Jim Sacchettini for continuing help and collegial input. This work was supported by the Life Sciences Task Force.
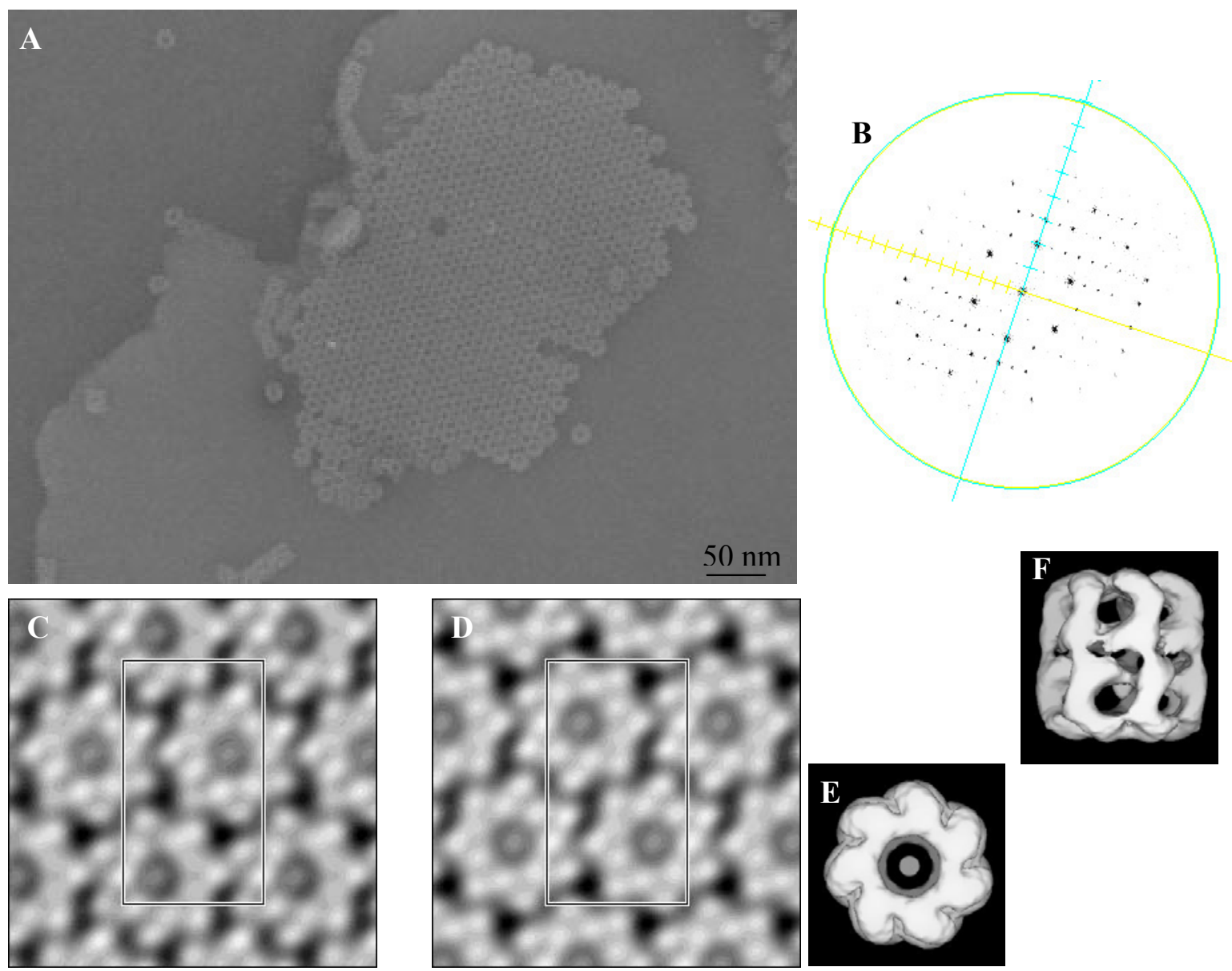

Fig 1. 2D crystallization of the GroEL-LacY complex using the lipid monolayer technique.(A) Electron micrograph of a negatively stained (1\% w/v uranyl acetate, $\mathrm{pH} 4.25)$. (B) FFT of the crystal $\left(\mathrm{a} \mathrm{x} \mathrm{b}=14.2 \times 24.7 \mathrm{~nm}, \gamma=90^{\circ}\right)$ and Fourier projection maps calculated in $\mathrm{p} 1(\mathrm{C})$ and $\mathrm{p} 2$ (D). 3D reconstruction following single particle analysis viewed face-on (E) and side-on (F). 